\title{
Der Übergang von der Intensiv- auf die Normalstation und die Zeit danach. Eine qualitative Studie aus Sicht der Betroffenen und deren Angehörigen
}

\author{
Tamara Großbichler $^{1,2} \cdot$ Martin Nagl-Cupal ${ }^{2}$ \\ Eingegangen: 12. Februar 2019 / Angenommen: 25. Februar 2019 / Online publiziert: 14. März 2019 \\ (c) Der/die Autor(en) 2019
}

\section{Zusammenfassung}

Hintergrund Wenn sich Intensivpatienten von ihrem kritischen Krankheitszustand erholen, werden diese in der Regel auf eine Normalstation transferiert. Dies kann für sie als auch für ihre Angehörige mit großen Herausforderungen verbunden sein.

Zielstellung Ziel dieser Forschungsarbeit war es, zu beschreiben und zu verstehen, wie ehemalige, schwer kritisch kranke Intensivpatienten und deren Angehörige die Transferierung von der Intensiv- auf die Normalstation und die anschließende Entlassung nach Hause erleben.

Methodik Dieser Studie liegt ein qualitativer Forschungsansatz zugrunde. Die Datenerhebung erfolgte mittels qualitativen, leitfadengestützten Interviews mit Betroffenen und deren Angehörigen. Die Datenanalyse erfolgte mittels offenem und axialem Kodieren der Grounded Theory nach Strauss und Corbin.

Ergebnisse Das Erleben der Transferierung und die Zeit danach können, angeschlossen an eine Vorphase, als Prozess in 3 Phasen beschrieben werden: 1. Phase: Ankommen - Landen, 2. Phase: Dort sein - Aktiv werden, 3. Phase: Zurück ins Leben - Ablösung. Dieses Phasenmodell zeigt auf, welche Veränderungen durch den gemeinsam durchlebten Prozess in Bezug auf Interaktionen, Rollen und Beziehungen stattfinden. Beide erleben die Zeit unmittelbar nach der Transferierung als Bruch, der zu Enttäuschung und Frustration führt. Die Angehörigen versuchen, die vorhandenen Lücken auszugleichen, indem sie aktiv eingreifen und eine tragende Rolle einnehmen.

Diskussion/Schlussfolgerungen Die Ergebnisse zeigen, dass Betroffene und Angehörige sowohl im Zusammenhang mit dem Übergang als auch in der Zeit danach mit verschiedenen Herausforderungen zu kämpfen haben und dabei meist keine adäquate Unterstützung erhalten. Für beide Gruppen ist eine kontinuierliche Begleitung vor, während und nach dem Transferprozess und auch zu Beginn auf der Normalstation eine Notwendigkeit.

Schlüsselwörter Kritisch kranker Patient $\cdot$ Transfer $\cdot$ Angehörige $\cdot$ Erleben

\section{The transition from the intensive care unit to the normal ward and the time beyond. A qualitative study from the perspective of patients and their relatives}

\section{Abstract}

Background As soon as patients have recovered from a critical illness in the intensive care unit (ICU) they are usually transferred to a normal ward for further care. The transfer represents an important step towards recovery but the transition itself and the time afterwards may be perceived as major challenges for both patients and their relatives.

Aus Gründen der besseren Lesbarkeit wird in diesem Beitrag überwiegend das generische Maskulinum verwendet. Dies impliziert immer beide Formen, schließt also die weibliche Form mit ein.

Tamara Großbichler tamara.groszbichler@wienkav.at
Martin Nagl-Cupal

martin.nagl-cupal@univie.ac.at

Anzengrubergasse 12/31, 1050 Wien, Österreich

Institut für Pflegewissenschaft, Universität Wien, Alser Straße 23/12, 1080 Wien, Österreich 
Aim The aim of this study was to describe and understand how former ICU patients and their relatives experienced the transfer from the ICU to the normal ward and the time beyond until discharge.

Method Data were collected using qualitative, semi-structured interviews with both patients and their relatives. Data analysis was based on the grounded theory approach of Strauss and Corbin.

Results Following a preliminary phase the transfer and the time on the normal ward can be described as a process in three phases. Phase 1: arrival - landing, phase 2: being there - becoming active, phase 3: coming back to life - taking off. This process shows the changes in interaction, the roles and the relationship between patients and their relatives. At the beginning patients and relatives experience the transition as a turning point that leads to disappointment and frustration. If necessary, relatives do their best to fill the gaps in order to care for the patient by actively intervening and undertaking roles left open by the staff.

Discussion and conclusion The results show that both patients and relatives struggle with various challenges associated with the transition and the time afterwards due to the perceived inadequate support. Continuous accompaniment during and after the transfer process and also at the beginning of the time on the normal ward is a necessity for patients as well as their relatives.

Keywords Critically ill patients $\cdot$ Transition $\cdot$ Relatives $\cdot$ Experience

\section{Einleitung und Problemdarstellung}

In Österreich werden jährlich ca. 150.000 Patienten auf einer Intensivstation betreut (Bundesministerium für Gesundheit 2012). Die Behandlung während des Intensivaufenthaltes ist für viele Patienten und Angehörige mit Stress und Ungewissheit verbunden (Duffield et al. 2011; Rotondi et al. 2002). Haben sich die Betroffenen von ihrem kritischen Zustand so weit erholt, dass sie die intensivmedizinische Betreuung nicht mehr benötigen, werden sie auf eine Normalstation zur weiteren Behandlung transferiert. Sowohl für die Ärzte als auch für das Pflegepersonal ist eine Transferierung ein Routinevorgang, und somit wird ihr nur wenig Aufmerksamkeit geschenkt (Mayer 2002).

Für die Patienten stellt die Transferierung einen wichtigen Schritt in Richtung Genesung dar. Sie ist aber auch mit verschiedenen negativen Gefühlen verbunden. Der Patient verlässt eine gut bekannte Umgebung, mit einem vertrauten Team, und wird in der Folge mit fremdem Personal und einer anderen Umgebung konfrontiert. Damit können psychische Probleme wie Angst, „relocation stress“ oder ein posttraumatisches Stresssyndrom einhergehen (Coyle 2001; Ludin et al. 2013; McKinney und Deeny 2002). Ausschlaggebend für eine Transferierung von der Intensivstation auf die Normalstation ist nicht nur der verbesserte Gesundheitszustand, sondern auch der Bedarf an Intensivbetten. Da nur eine begrenzte Zahl an Intensivbetten für die Versorgung zur Verfügung steht, kann dieser Umstand einen Transfer der Patienten auf die Normalstation beschleunigen (Gibson 1997).

Angehörige können ebenfalls vom Transfer betroffen sein. Sie ängstigen sich v. a., weil ihre kranken Angehörigen von einem sehr gut überwachten Bereich der Intensivstation auf einen in ihren Augen weniger gut überwachten Bereich der Normalstation verlegt werden. Damit sind in der Folge ebenfalls negative Gefühle verbunden (Bokinskie 1992; Mitchell et al. 2003; Nagl-Cupal und Schnepp 2011).

Laut Wesson (1997) haben Angehörige einen hohen Einfluss auf die Genesung des Patienten. Durch die familiäre Unterstützung können Stress und Angst reduziert werden, und dies wirkt sich positiv auf die Gesundheit aus (Gibson 1997; Mayer 2002).

Die vorhandene Forschung zeigt, dass das Thema Transferierung von der Intensivstation vonseiten der Patienten und der Angehörigen bereits gut erforscht ist. Wenig bekannt ist, wie sich dieses Erleben auf ein und dieselbe Situation aufeinander bezieht und voneinander abweicht und sich über den gesamten Verlauf der stationären Pflege verändert. Dies ist deshalb relevant, weil Angehörige auf der Intensivstation und darüber hinaus häufig die zentralen Bezugspersonen sind, die im Sinne der Patienten sehr wichtige Aufgaben übernehmen. Besonders problematisch ist die Situation bei jenen Patienten, die aufgrund einer langwierigen kritischen Erkrankung sehr lange auf der Intensivstation gepflegt werden müssen. Sie haben nach der Intensivstation häufig noch einen hohen Pflegebedarf, und dies stellt auch Pflegende vor besondere Herausforderungen (Cox 2012; Duffield et al. 2011; Fink und Suter 2006; Lin et al. 2009; Whittaker und Ball 2000).

\section{Zielsetzung und Fragestellung}

Ziel der Forschungsarbeit ist es, Einblick in das Erleben der Transferierung ehemals kritisch kranker Intensivpatienten mit vorangegangem langem Intensivaufenthalt und ihrer Angehörigen zu geben, um mögliche Situationen zu identifizieren, an denen Betroffene Unterstützung benötigen. Daraus ergibt sich folgende Fragestellung: Wie erleben ehemals kritisch kranke Intensivpatienten und deren Angehö- 
rige den Übergang von der Intensiv- auf die Normalstation und die Zeit danach?

\section{Methodik}

Zur Beantwortung der Forschungsfrage wurde der qualitative Forschungsansatz gewählt, da sie den Anspruch hat, die Lebenswelt aus Sicht der Betroffenen zu beschreiben und somit zu einem besseren Verständnis der sozialen Wirklichkeit beiträgt (Flick et al. 2008). Zur Datenerhebung wurden leitfadengestützte Einzelinterviews geführt. Die zu erfüllenden Einschlusskriterien der an der Studie Teilnehmenden sahen wie folgt aus: Befragt wurden einerseits ehemalige sedierte, beatmete Intensivpatienten ab 18 Jahren, die von der Intensivstation auf die Normalstation verlegt wurden. Die befragten Patienten mussten einen Mindestaufenthalt von 7 Tagen auf der Intensivstation aufweisen. Ein weiteres Einschlusskriterium war, dass sich die zu Interviewenden sowohl an den Transfer von der Intensiv- auf die Normalstation als auch an die Zeit danach erinnern mussten. Die Probanden sollten in der Lage sein, ihre Erfahrungen mündlich, in deutscher Sprache, mitteilen zu können.

Andererseits wurden pro Patient ein Angehöriger befragt, der von dem Betroffenen selbst, nach dem Grad der Wichtigkeit der Anwesenheit im Hinblick auf die Zeit der Transferierung und danach, ausgewählt wurde. Diese mussten ebenfalls über 18 Jahre alt sein und in deutscher Spra- che ein Interview absolvieren können. Die Tab. 1 gibt einen detaillierten Überblick über die interviewten Patienten und deren Angehörige.

Die Rekrutierung der Teilnehmenden erfolgte über ein großes Krankenhaus und verschiedene Intensivstationen. $\mathrm{Da}$ es sich bei den Untersuchungspersonen um bereits entlassene Patienten handelte, erfolgte die Suche nach passenden Interviewpersonen mithilfe der elektronischen Dokumentation. Die Genehmigung dafür wurde im Vorfeld von der Pflegedirektion und dem Träger des Krankenhauses erteilt, weil die Forschende zum Zeitpunkt der Untersuchung in diesem Krankenhaus arbeitete. Da es sich um bereits entlassene Patienten handelte und aufgrund der vergleichsweise geringen Vulnerabilität, war in Absprache mit der zuständigen Ethikkommission kein ethisches Gutachten zwingend notwendig. Im Rahmen der Forschung wurden die Richtlinien gemäß der Helsinki-Deklaration eingehalten (World Medical Association 2013).

Nach persönlicher Kontaktaufnahme und Interessenbekundung erhielten die zu Befragenden ein detailliertes Informationsschreiben und wurden mündlich über Studienziele, Vorgehensweise, Freiwilligkeit, Datensicherheit und -anonymität aufgeklärt. Die informierte Zustimmung wurde von den Teilnehmenden in schriftlicher Form eingeholt. Das Sampling selber erfolgte mittels einer bewussten Auswahl von infrage kommenden Personen. Insgesamt wurden im Zeitraum Juli bis Oktober 201510 Interviews geführt (Tab. 1). Die Interviews dauerten im Schnitt $60 \mathrm{~min}$.

Tab. 1 Befragte Patienten und Angehörige

\begin{tabular}{|c|c|c|c|c|c|c|c|c|}
\hline $\begin{array}{l}\text { Interview- } \\
\text { person }\end{array}$ & Geschlecht & $\begin{array}{l}\text { Alter } \\
\text { (Jahre) }\end{array}$ & Beruf & $\begin{array}{l}\text { Aufnahme- } \\
\text { grund }\end{array}$ & $\begin{array}{l}\text { Intuba- } \\
\text { tions- } \\
\text { zeit }\end{array}$ & $\begin{array}{l}\text { Aufenthalt } \\
\text { auf der } \\
\text { ICU* }\end{array}$ & $\begin{array}{l}\text { Interventionen und } \\
\text { Probleme während } \\
\text { des ICU-Aufent- } \\
\text { halts }\end{array}$ & $\begin{array}{l}\text { Aufenthalt } \\
\text { auf der } \\
\text { Normal- } \\
\text { station }\end{array}$ \\
\hline Patient 1 & Männlich & 66 & $\begin{array}{l}\text { Pensionist; früher } \\
\text { Schlosser }\end{array}$ & $\begin{array}{l}\text { Rupturiertes } \\
\text { Bauch- } \\
\text { aortenan- } \\
\text { eurysma }\end{array}$ & $\begin{array}{l}\text { Mehr als } \\
7 \text { Tage }\end{array}$ & 21 Tage & $\begin{array}{l}\text { Diverse Komorbidi- } \\
\text { täten } \\
\text { Zäkumperforation, } \\
\text { Peritonitis, SIRS } \\
\text { Pleurapunktion }\end{array}$ & 12 Tage \\
\hline $\begin{array}{l}\text { Ehefrau } \\
\text { von } \mathrm{Pa}- \\
\text { tient } 1\end{array}$ & Weiblich & 63 & $\begin{array}{l}\text { Pensionistin; früher } \\
\text { kaufmännische } \\
\text { Angestellte }\end{array}$ & - & & & & \\
\hline Patient 2 & Männlich & 69 & $\begin{array}{l}\text { Pensionist; früher } \\
\text { Ingenieur bei einer } \\
\text { internationalen } \\
\text { Firma }\end{array}$ & $\begin{array}{l}\text { Polytrauma } \\
\text { nach } \\
\text { Verkehrs- } \\
\text { unfall }\end{array}$ & $\begin{array}{l}\text { Mehr als } \\
7 \text { Tage }\end{array}$ & 32 Tage & $\begin{array}{l}\text { Isolation in Ein- } \\
\text { zelzimmer wegen } \\
\text { Problemkeim } \\
\text { Rotationstherapie } \\
\text { aufgrund des } \\
\text { schweren Ver- } \\
\text { letzungsmusters } \\
\text { notwendig } \\
\text { Tracheotomie } \\
\text { Schluck- und } \\
\text { Sprachstörungen }\end{array}$ & 30 Tage \\
\hline $\begin{array}{l}\text { Ehefrau } \\
\text { von Pa- } \\
\text { tient } 2\end{array}$ & Weiblich & 64 & $\begin{array}{l}\text { Professorin für } \\
\text { Biochemie }\end{array}$ & - & & & & \\
\hline
\end{tabular}


Tab. 1 (Fortsetzung)

\begin{tabular}{|c|c|c|c|c|c|c|c|c|}
\hline $\begin{array}{l}\text { Interview- } \\
\text { person }\end{array}$ & Geschlecht & $\begin{array}{l}\text { Alter } \\
\text { (Jahre) }\end{array}$ & Beruf & $\begin{array}{l}\text { Aufnahme- } \\
\text { grund }\end{array}$ & $\begin{array}{l}\text { Intuba- } \\
\text { tions- } \\
\text { zeit }\end{array}$ & $\begin{array}{l}\text { Aufenthalt } \\
\text { auf der } \\
\text { ICU* }\end{array}$ & $\begin{array}{l}\text { Interventionen und } \\
\text { Probleme während } \\
\text { des ICU-Aufent- } \\
\text { halts }\end{array}$ & $\begin{array}{l}\text { Aufenthalt } \\
\text { auf der } \\
\text { Normal- } \\
\text { station }\end{array}$ \\
\hline \multirow[t]{4}{*}{ Patientin 3} & \multirow[t]{4}{*}{ Weiblich } & \multirow[t]{4}{*}{56} & \multirow{4}{*}{$\begin{array}{l}\text { Buchhalterin; } \\
\text { derzeit im } \\
\text { Krankenstand }\end{array}$} & \multirow{4}{*}{$\begin{array}{l}\text { Polytrauma } \\
\text { nach } \\
\text { Verkehrs- } \\
\text { unfall }\end{array}$} & \multirow[t]{4}{*}{$\begin{array}{l}3 \text { Tage } \\
\text { intubiert }\end{array}$} & \multirow[t]{4}{*}{13 Tage } & $\begin{array}{l}\text { Komplexe Verlet- } \\
\text { zungen }\end{array}$ & \multirow[t]{4}{*}{74 Tage } \\
\hline & & & & & & & $\begin{array}{l}\text { Zwei Operationen } \\
\text { während des ICU- } \\
\text { Aufenthalts }\end{array}$ & \\
\hline & & & & & & & Pleurapunktion & \\
\hline & & & & & & & $\begin{array}{l}\text { NIV-Therapie not- } \\
\text { wendig }\end{array}$ & \\
\hline $\begin{array}{l}\text { Ehemann } \\
\text { von Pati- } \\
\text { entin } 3\end{array}$ & Männlich & 65 & $\begin{array}{l}\text { Pensionist; früher } \\
\text { Koch }\end{array}$ & - & & & & \\
\hline \multirow[t]{4}{*}{ Patientin 4} & \multirow[t]{4}{*}{ Weiblich } & \multirow[t]{4}{*}{59} & \multirow[t]{4}{*}{$\begin{array}{l}\text { Pensionistin; früher } \\
\text { Raumpflegerin }\end{array}$} & \multirow{4}{*}{$\begin{array}{l}\text { Geplante } \\
\text { Operation } \\
\text { eines sub- } \\
\text { renalen } \\
\text { Aortenan- } \\
\text { eurysmas }\end{array}$} & $\begin{array}{l}\text { Mehr als } \\
7 \text { Tage }\end{array}$ & \multirow[t]{4}{*}{30 Tage } & $\begin{array}{l}\text { Diverse Komorbidi- } \\
\text { täten }\end{array}$ & \multirow[t]{4}{*}{33 Tage } \\
\hline & & & & & & & $\begin{array}{l}\text { Dialyse während } \\
\text { des ICU-Aufent- } \\
\text { halts }\end{array}$ & \\
\hline & & & & & & & $\begin{array}{l}\text { Schmerzen und } \\
\text { Schluckprobleme }\end{array}$ & \\
\hline & & & & & & & $\begin{array}{l}\text { NIV-Therapie not- } \\
\text { wendig }\end{array}$ & \\
\hline $\begin{array}{l}\text { Ehemann } \\
\text { von Pati- } \\
\text { entin } 4\end{array}$ & Männlich & 58 & $\begin{array}{l}\text { Pensionist; früher } \\
\text { Feuerwehmann }\end{array}$ & - & & & & \\
\hline \multirow[t]{4}{*}{ Patient 5} & \multirow[t]{4}{*}{ Männlich } & \multirow[t]{4}{*}{62} & \multirow[t]{4}{*}{$\begin{array}{l}\text { Pensionist; früher } \\
\text { Gießereitechniker }\end{array}$} & \multirow{4}{*}{$\begin{array}{l}\text { Rupturiertes } \\
\text { Aneurys- } \\
\text { ma der } \\
\text { Aorta } \\
\text { thoracica }\end{array}$} & $\begin{array}{l}\text { Mehr als } \\
7 \text { Tage }\end{array}$ & \multirow[t]{4}{*}{31 Tage } & $\begin{array}{l}\text { Diverse Komorbidi- } \\
\text { täten }\end{array}$ & \multirow[t]{4}{*}{69 Tage } \\
\hline & & & & & & & Reanimation & \\
\hline & & & & & & & $\begin{array}{l}\text { Dialysepflichtig } \\
\text { während ICU-Auf- } \\
\text { enthalts bis jetzt }\end{array}$ & \\
\hline & & & & & & & $\begin{array}{l}\text { NIV-Therapie not- } \\
\text { wendig }\end{array}$ & \\
\hline $\begin{array}{l}\text { Ehefrau } \\
\text { von Pa- } \\
\text { tient } 5\end{array}$ & Weiblich & 72 & $\begin{array}{l}\text { Pensionistin; früher } \\
\text { Hausfrau }\end{array}$ & - & & & & \\
\hline
\end{tabular}

ICU* „,intensive care unit“; SIRS „systemic inflammatory response syndrome“, NIV „noninvasive ventilation“

Die Datenauswertung erfolgte mittels offenen und axialen Kodierens des Analyseverfahrens der Grounded Theory nach Strauss und Corbin (Strauss und Corbin 1996). Im ersten Schritt, dem offenen Kodieren, wurden Kodes und Kategorien durch eine Zeile-für-Zeile-Analyse erzeugt, und bedeutsame Textstücke wurden im Zuge der Datenauswertung mit In-vivo-Kodes versehen. Im zweiten Schritt, dem axialen Kodieren, wurden die zuvor aufgebrochenen Daten auf neue Art und Weise wieder zusammengesetzt. Dies wurde mithilfe eines paradigmatischen Modells (Kodierparadigmas) erreicht (Strauss und Corbin 1996). Das paradigmatische Modell bildet die Grundlage des entwickelten Phasenmodelles.

\section{Ergebnisse}

Das Erleben des Übergangs auf die Normalstation und wieder nach Hause kann sowohl bei kritisch kranken Intensivpatienten als auch bei deren Angehörigen als Verlauf in 4 Phasen dargestellt werden (Abb. 1). Es zeigt sich auch, dass mit der Transferierung auf die Normalstation der Versuch der Wiedergewinnung von Normalität und Kontrolle nicht abgeschlossen ist.

Den 3 Hauptphasen vorgelagert ist eine Vorphase „Bis es wieder bergauf geht und Ordnung ins Chaos bringen", die die Erlebnisse und Erfahrungen der Betroffenen und ihrer Angehörigen bis zum Zeitpunkt des Transfers beschreibt. Diese Phase hat Einfluss darauf, wie die weitere Zeit erlebt und bewertet wird. Die erste Phase „Ankommen und Landen“ beginnt mit dem Übergang von der Intensiv- auf die 
Abb. 1 Eigene Darstellung des Phasenmodells aus Patienten und Angehörigensicht
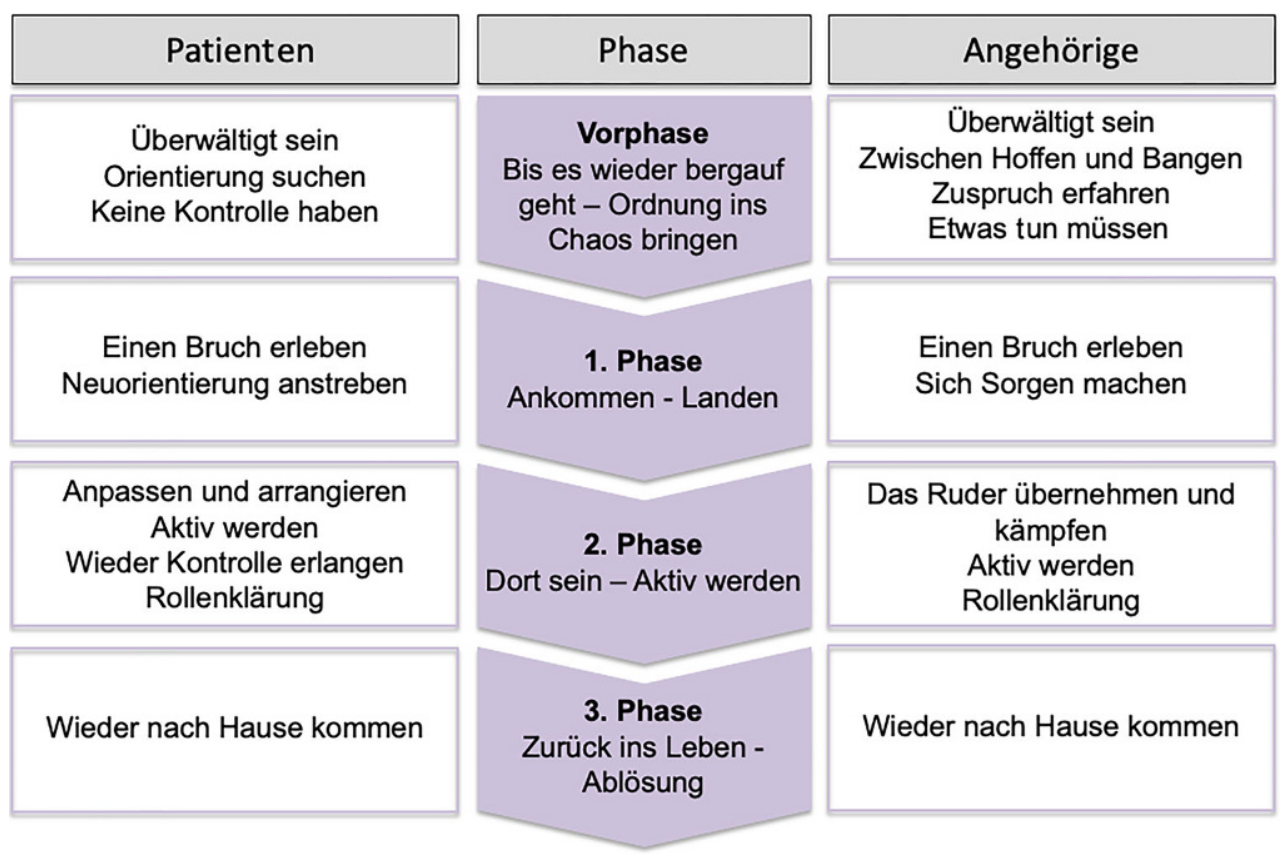

Normalstation und bezieht sich auf die ersten paar Tage auf der Normalstation. Die zweite Phase „Dort sein und aktiv werden" umfasst die Erfahrungen der Patienten und ihrer Angehörigen ein paar Tage nach Ankunft auf der Normalstation bis kurz vor Ende des Aufenthalts im Krankenhaus. Die dritte und letzte Phase ,Zurück ins Leben und Ablösung" beschreibt die Erfahrung der Entlassung nach Hause oder in eine andere Versorgungseinrichtung wie z.B. eine Rehaklinik. Die Hauptkategorie jeder Phase (Abb. 1) ist sowohl für die Betroffenen als auch ihre Angehörigen gleich und stellt einen Rahmen für die teilweise unterschiedlichen Subkategorien der Patienten einerseits und deren Angehörigen andererseits dar. Obwohl sich die Phasen modellhaft zeitlich begrenzt darstellen lassen, pendeln manche Patienten und deren Angehörige zwischen diesen Phasen hin und her und befinden sich nicht immer zur gleichen Zeit in derselben Phase. In weiterer Folge werden die unterschiedlichen Phasen aus Patientensicht sowie aus Angehörigensicht näher beleuchtet.

\section{Vorphase: Bis es wieder bergauf geht und Ordnung ins Chaos bringen}

„Bis es wieder bergauf geht und Ordnung ins Chaos bringen" stellt die Hauptkategorie sowohl für Patienten als auch ihre Angehörigen in dieser Phase dar. Sie beginnt bei den Patienten mit dem ersten Erwachen auf der Intensivstation. Viele Betroffene können sich weder an Unfallhergänge oder an akute Geschehnisse, die zur Aufnahme auf die Intensivstation geführt haben, erinnern. Sie sind überwältigt, finden sich in einer unbekannten Umgebung wieder, umge- ben von fremden Personen, die sie nicht zuordnen können. Sie durchleben häufig Albträume, und es fällt ihnen schwer, Realität von Geträumtem zu unterscheiden. Für viele Betroffene ist dies belastend, weil sie schlimme Erlebnisse auf der Intensivstation nicht einordnen und daher nur schwer damit umgehen können. Es ist für sie wichtig, dass sie Orientierung suchen, was länger dauern und sogar über die Intensivstationszeit hinausgehen kann, wie ein ehemaliger Patient beschreibt.

Nur da habe ich wie gesagt nicht einmal noch realisiert gehabt, was eigentlich los ist, dass ich eben nicht aufstehen kann. [...] Also ich habe es ja nicht einmal richtig registriert, dass ich eben jetzt übersiedelt werde. (Patientin 3)

Da die Patienten in der Vorphase in der Regel keine Kontrolle über sich selbst haben, schätzen viele rückblickend die Intensivstationszeit als ,gefährlich“ ein, da es aus ihrer Sicht jederzeit zu einer Verschlechterung des Gesundheitszustandes kommen konnte. Aufgrund dieser wahrgenommenen latenten Gefahr werden Angehörige von den Betroffenen als essenzielle Stütze erlebt:

[...] weil du wirklich, also schwerst bedient bist (..) und man nie genau sagen konnte, gibt es eine Komplikation oder nicht. Ich habe mich gefreut, ich habe meine Frau gespürt, das weiß ich (,) weiß ich noch. (Patient 2)

Gute pflegerische Betreuung und eine Beziehung zu den Pflegenden auf der Intensivstation werden von Patienten positiv wahrgenommen und haben für die Betroffenen rückblickend einen hohen Stellenwert. Sie haben das Potenzial, 
Orientierung zu geben und dadurch die „Schattenseiten“ der Intensivstation in Form von Schlaflosigkeit, Albträumen und Ängsten zu kompensieren:

[...], das war immer vom ersten Moment [lacht], wie ich wieder munter geworden bin, das war immer (..), das war immer gut. Das alleine hilft schon, dass man wieder auf den Damm kommt. (Patient 2)

Bei den Angehörigen beginnt diese Vorphase bereits mit der Aufnahme des Patienten auf die Intensivstation. Bei einem ungeplanten Intensivaufenthalt, durch einen Unfall oder eine akute Operation, verändert sich das Leben der Angehörigen von heute auf morgen. Sie sind überwältigt von der Umgebung und dem technischen Equipment der Intensivstation, durchleben verschiedenste Gefühle wie Angst, Schock, Sorge und Ungewissheit und pendeln dabei zwischen Hoffen und Bangen. In dieser Situation ist es für die Angehörigen substanziell, dass sie vom behandelnden Team häufig Zuwendung und Zuspruch erfahren, um diese ungewisse Zeit leichter meistern zu können und eine Vertrauensbasis zwischen den Angehörigen und dem Team zu schaffen.

Trotz der erlebten Überforderung haben Angehörige stets das Verlangen, für ihre kranken Angehörigen etwas tun zu müssen. Die Angehörigen versuchen, immer da zu sein, solange es die Besuchszeiten zulassen.

$\mathrm{Da}$, da tut man irgendwas, dann sitzt, dann plaudert man, [...] da hat sie mich wahrscheinlich noch gar nicht richtig verstanden, da habe ich auch geplaudert mit ihr, da bin ich da drinnen gesessen alleine da in dem Intensivraum (,) und habe ihr halt den Tagesablauf erzählt und so. Also, obwohl sie mich vielleicht gar nicht (,) so richtig verstanden hat, nicht. (Ehemann von Patientin 3)

Erkennen die Angehörigen erste Zeichen einer Besserung oder erhalten sie gute Nachrichten, so erleben sie ein Gefühl der Freude, Erleichterung und nehmen an, dass es bergauf gehe. Dieses Gefühl wird durch den Transfer auf die Normalstation bestärkt, der die nächste Phase einleitet.

\section{Erste Phase: Ankommen - Landen}

Diese Phase bezieht sich auf den Übergang von der Intensiv- auf die Normalstation und die ersten paar Tage nach dem Ankommen auf der Normalstation. Obwohl der Transfer grundsätzlich positiv bewertet wird, ist es trotzdem so, dass Patienten nach dem Transfer häufig einen Bruch erleben, der zu Enttäuschung und Frustration führt. Dieser Bruch ist z.T. der Verschlechterung der Umgebung, z.B. durch Mehrbettzimmer geschuldet, aber auch der erlebten geringeren Betreuungsintensität. Diese führt nicht selten zu einer Verschlechterung des Gesundheitszustandes und steht damit im Widerspruch zu der mit der Transferierung assoziierten gesundheitlichen Verbesserung. Dies führt häufig wiederum dazu, dass der Beziehungsaufbau zu den Pflegenden gestört wird und Patienten sich alleine fühlen. Manche beschreiben die wahrgenommene Verschlechterung der pflegerischen Betreuung sogar als Schock:

Das war momentan ein Schock für mich, weil (..) so wie friss oder stirb, so ist das gewesen dort, so ist mir das dort vorgekommen. (Patient 1).

Dabei erleben sie auch die Forderung nach einer gewissen Selbstständigkeit, wofür sie sich u. U. noch nicht bereit fühlen. Die Übernahme von Eigenverantwortung wird in diesem Fall als von außen oktroyiert wahrgenommen und kann zu Überforderung und Enttäuschung führen:

[...] wenn du da gerade von der Intensivstation raufkommst, wenn du eh noch nicht mobil bist, wo du eh unsicher bist, ob du gehst oder aufstehst oder irgendetwas, dann ist das schon ein bisschen nicht so das Richtige, sage ich halt so; nicht. Es war halt schon (..) nicht zufriedenstellend. (Patient 1).

Im Falle einer gesundheitlichen Verschlechterung benötigen in dieser Phase die Betroffenen verstärkt Unterstützung von den Angehörigen. Diese übernehmen nun die Rolle eines Anwalts bzw. eines Fürsprechers und befinden sich selbst, wenn sie diese Rolle wahrnehmen, schon in Phase zwei. Patienten erleben ihre Angehörigen in einer neuen Rolle, die auf der Intensivstation bisher nicht notwendig war:

[...] hat hier und da schon ein bisschen Dampf machen müssen (..), was auf der Intensiv überhaupt nie zur Diskussion gestanden ist. Das war einfach kein, (,), kein Punkt. (Ehefrau von Patient 2).

Die Patienten versuchen, sich in der neuen Umgebung zurechtzufinden und eine Neuorientierung anzustreben. Der Übergang der Betroffenen von einer passiven in eine aktive Rolle beginnt meist ein paar Tage nach der Ankunft auf der Normalstation, wenn die ersten Eindrücke, Erlebnisse und Enttäuschungen überwunden sind. Dieser Übergang führt in die zweite Phase.

Parallel dazu beschreiben Angehörige die Zeit nach der Transferierung ebenfalls als einen Bruch, gekennzeichnet durch Enttäuschung oder Frustration. Sie machen sich Sorgen um ihre kranken Angehörigen. Sie helfen ihren kranken Angehörigen und sind bereit einzugreifen, wenn es notwendig ist.

Ich habe gesagt, das sehe ich jetzt nicht ein. Er wird nicht operiert, er lungert da hinten, der bekommt nur Krankheiten; eine nach der anderen. Jeden Tag, wenn du da rein gekommen bist, hast du schon Angst ge- 
habt, was er jetzt hat. (,) und eben das Seelische war auch. (Ehefrau von Patient 5)

Sie versuchen, die Anliegen und Bedürfnisse der bzw. des kranken Angehörigen durchzusetzen, was häufig als schwieriger und anstrengender „Kampf“ erlebt wird. Diesem Kampf widmen sie ihre ganze Energie und wachsen z.T. über sich hinaus, weil sie sich verantwortlich fühlen. Diese Aktivität führt die Angehörige bzw. den Angehörigen dann in Phase zwei.

\section{Zweite Phase: Dort sein - aktiv werden}

Die zweite Phase und das Dort sein aus der Sicht der Patienten bezieht sich auf deren Erfahrungen, nachdem sie auf der Normalstation angefangen haben, Eigenverantwortung zu übernehmen und reicht bis kurz vors Ende des Aufenthalts im Krankenhaus. Die Patienten bemühen sich, auf unterschiedliche Art und Weise so schnell wie möglich, mit den erlebten Enttäuschungen aus Phase eins umzugehen und mit den vorherrschenden Gegebenheiten zurechtzukommen und sich mit diesen zu arrangieren. Sie nehmen es einfach hin, weil sie es nicht ändern können oder sie schrauben die Ansprüche an ihre eigene Betreuung immer weiter zurück:

Man muss das halt hinnehmen wie es ist, das kannst du eh nicht ändern. (..) Wissen Sie, das mit dem laut Schlafen, Schnarchen [lachte] ist ja auch nicht einfach (..). Aber an und für sich ist es eh gegangen. Mit den Leuten habe ich halt ein Glück gehabt, es ist halbwegs gegangen, ja, ja, halbwegs normale Leute. Es ist schon gegangen ja, naja (..). (Patient 1).

An die Anpassung schließt in der Regel die Zeit an, in der sie wieder aktiv werden. Die geht mit der zunehmenden gesundheitlichen Genesung einher. Viele Betroffene erleben diese Zeit als anstrengend, ähnlich einem Kampf, weil auch Einfaches, wie das Anhalten an einem Griff, um aus dem Rollstuhl in die Dusche zu kommen, als massiver Kraftakt erlebt wird. Bestimmt wird diese Zeit von ihrer Hartnäckigkeit im Erreichen des Ziels, wieder nach Hause zu kommen. Somit fällt es ihnen leichter, die damit einhergehenden Strapazen durchzustehen:

Das habe ich können selber. Das habe ich auch gar nicht wollen, weil das, ich habe eh Zeit gehabt. [...] Ich wollte das selber nein weil [lacht] (..) Nein, weil, so fremde Hilfe, das habe ich eh lange genug auf der Intensiv gebraucht. (Patientin 4).

Die Kontrolle wieder zu übernehmen, kann auf den Körper beschränkt bleiben, und Entscheidungen oder die Kommunikation mit dem medizinischen Personal werden teilweise oder auch komplett ihren Angehörigen überlassen, es kann aber auch viel umfassender sein, wenn manche Patienten aufgrund psychischer und/oder physischer Verschlechterung und Motivationsverlustes ihre bereits zurückgewonnene Kontrolle wieder abgeben, wie z.B. das Gehen mit dem Rollator einstellen und sich zurückziehen, und die Angehörigen dann an deren Stelle das Ruder übernehmen. Im Zuge des Wiedererlangens von Kontrolle kommt es auch zum Ausverhandeln von Rollen bzw. zur Rollenklärung zwischen den Betroffenen und ihren Angehörigen, um den Prozess der Genesung gemeinsam aktiv gestalten zu können, sei es explizit oder implizit.

Aufgrund der Erfahrung aus Phase eins versuchen die Angehörigen, alles zu tun, dass die Situation auf der Normalstation sowohl für sie als auch für den Patienten erträglicher wird, indem sie aktiv am Genesungsprozess und am Ablauf dort eingreifen. Sie übernehmen das Ruder und versuchen, falls notwendig, sich sofort um eine gesundheitliche Verbesserung des Patienten zu bemühen. Des Öfteren erleben Angehörige aber Macht- und Hilflosigkeit, weil sie ohne Unterstützung nichts erreichen können. Somit sehen sich manche gezwungen, auf ,externe Hilfe“ zurückzugreifen, weil sich Frustration einstellt und sie vielleicht auch keine Kraft mehr haben alleine weiterzukämpfen:

Die haben einfach nicht reagiert, bis ich dann die Betriebsärztin meines Mannes angerufen habe, ihr die Situation geschildert habe und die dann mal endlich dort aufgetreten ist [...]. (Ehefrau von Patient 2).

Die Angehörigen erleben sich meist in mehreren unterschiedlichen Rollen, die sie ausüben, so lange es aus ihrer Sicht notwendig ist.

\section{Dritte Phase: Zurück ins Leben - Ablösung}

In dieser Phase finden die Patienten wieder zurück ins Leben, und es kommt zu einer Ablösung aus dem klinischen Setting hin zum gewohnten Umfeld. Die Phase bzw. das Wieder-nach-Hause-Kommen wünschen sich sowohl die Patienten als auch die Angehörigen schon früher herbei, als dies dann tatsächlich eintritt, und das Hoffen ist bei den Patienten immer präsent. Die schweren, kritischen Zeiten sind überstanden. Die Betroffenen versuchen, gemeinsam mit der ihm bzw. ihr vertrauten Person dort wieder ein normales, geregeltes Leben zu führen. Die Angehörigen nehmen sich nun wieder mehr Zeit für sich, weil der Patient endlich zu Hause ist und dieser Zustand es eben möglich macht, was vorher nicht der Fall war.

Die Beziehung zwischen Patient und vertrauter Person zu Hause kann zu Beginn mit neuen Herausforderungen konfrontiert werden. Diese können durch die Folgeerscheinungen der schweren Erkrankung verursacht werden, welche von möglicherweise weiterhin bestehenden körperli- 
chen Einschränkungen bis hin zur psychischen Verarbeitung dieser schweren Zeit reichen. Somit ist auch zu Hause der Prozess der Genesung nicht abgeschlossen, weil immer eine Erinnerung an diese erlebte Zeit bleibt, wie folgendes Zitat beschreibt:

Ja, na das ist ein langer Prozess. Wie gesagt (,) die Dialyse und alles, das ist noch nicht gerade lustig. [...]

Weil man nichts machen kann, der Tag ist verloren. (Patient 5).

Die Angehörigen sind genauso wie die Betroffenen dazu bereit, vorübergehende Probleme und die damit verbunden Tätigkeiten wie z.B. Verabreichung von Sondennahrung, selbstständig zu Hause durchzuführen. Aus ihrer Sicht bedarf es keiner externen Unterstützung mehr, und sie sind überzeugt davon, dass sie das gemeinsam gut schaffen:

[...] weil man jetzt, also die meisten Sachen so waren, dass man das auch zu Hause, also das mit der (..) Umstellung der Nahrung auf die PEG-Sonde dann, das hat er eh auch im Griff gehabt, und dann haben wir eigentlich das Gefühl gehabt: naja, das können wir zu Hause auch alles machen, das ist kein Problem. (Ehefrau von Patient 2).

Wenn die Betroffenen weitere Fortschritte machen, gibt das den Angehörigen Hoffnung und Kraft, dass es auch zu Hause gut funktionieren kann:

Ich war nur froh, dass er wieder zu Hause ist und dann Fortschritte gemacht hat, und wirklich, dass er so gekämpft hat. Ja, das war schon wichtig. (Ehefrau von Patient 1).

Die erlebte Zeit und der durchgemachte Prozess, sind für alle Patienten prägend. Sie sind froh und glücklich, die Zeit überstanden bzw. überlebt zu haben, und freuen sich, wieder im Kreise ihrer Familie und Freunde leben zu können. Zurückblickend auf den Prozess äußert ein Patient Folgendes:

[...] aber es ist auch sehr, ich glaube eine wichtige Lebenserfahrung, wenn man sie macht. [...] Wenn es einem nicht erspart bleibt [lacht] und weil man dadurch natürlich sehr viel lernt in verschiedener (Hinsicht?), erstens sich selber. (Patient 2).

\section{Diskussion}

Die Ergebnisse geben Einblick in das Erleben Betroffener und deren Angehöriger, wenn kritisch kranke Menschen von der Intensivstation auf die Normalstation und nach Hause transferiert werden.
Unter transitionstheoretischen Gesichtspunkten lassen sich dabei verschiedene Arten von Übergangen darstellen (Meleis 2010). Einerseits gesundheits- bzw. krankheitsbedingte Transition, also vom Überleben auf der Intensivstation hin zur Genesung. Andererseits parallel dazu eine durch den Setting-Wechsel situationsbedingte Transition, also aus Betroffenensicht von einem hochtechnisierten, individualisierten Umfeld, einer sicheren und vertrauten Umgebung hin zu einer unvorhersehbaren, verletzenden und von Abhängigkeit geprägten Umgebung (McKinney und Deeny 2002).

Vor allem zeigt sich aber, dass die Angehörigen neben dieser situationsbedingten Transition eine weitere Transition durchleben, was ihre Rolle als Angehörige bzw. Angehöriger betrifft. Auch Agard et al. (2015) konnte zeigen, dass Angehörige verschiedene Rollen bei der Genesung ehemaliger Intensivpatienten zu Hause einnehmen, vom Partner hin zur Pflegeperson und nach der Genesung wieder zurück. In der vorliegenden Arbeit zeigt sich darüber hinaus, dass Angehörige auch schon im Krankenhaus einen wichtigen Beitrag im Hinblick auf die Genesung leisten, indem sie bereits dort unterschiedliche Rollen wahrnehmen. Welche das sind, ist einerseits abhängig von deren Persönlichkeit, ihren Lebensumständen und Vorerfahrungen, andererseits aber auch davon, was sie imstande sind, in schwierigen Zeiten zu leisten bzw. sich aufzubürden. Es zeigte sich, dass die Angehörigen eine wichtige Rolle als „Orientierungsgeber“ einnehmen. Trotz der Erinnerung an den Transfer hatten Betroffene Probleme, sich zu orientieren und sich aktiv in den Genesungsprozess zu involvieren. Ohne Bewusstsein und Erinnerung an die Zeit auf der Intensivstation involvieren sich Patienten nicht (Ludin et al. 2013). Somit ist, Orientierung und Information zu geben und zu bekommen, für den weiteren Genesungsprozess wichtig, in dem Angehörige auch die Rolle der aktiv Unterstützenden einnehmen, um zu helfen und zu motivieren, wenn die kranken Angehörigen frustriert, ungeduldig sind oder an körperliche Grenzen kommen.

Ein großes Problem für die Betroffenen ist ihre Abhängigkeit auch in banalen Dingen, wie z.B. selbst nicht essen zu können, was demotivierend wirkt (Ramsay et al. 2013). In dieser Studie kann diesbezüglich ergänzt werden, dass Pflegende eine entscheidende Rolle dabei spielen, wie die Betroffenen diese Abhängigkeit erleben. In den Ergebnissen zeigt sich, dass Patienten aufgrund der Erwartungen des Pflegepersonals, selbstständig zu sein, überfordert sind. Auch in dieser Situation stehen Angehörige unterstützend zur Seite, um die Unselbstständigkeit zu kompensieren.

Die Patienten nehmen diese Rollenänderung nicht oder nur teilweise wahr, möglicherweise, weil sie es als eine Art Pflicht der Angehörigen ansehen, dass diese zusätzliche Aufgaben wahrnehmen, oder sie sind zu sehr mit ihrer eigenen Situation beschäftigt. 
In der Studie konnten auch Faktoren identifiziert werden, die sich hemmend auf einen Übergang auswirken können. Dies sind allen voran Veränderungen in der Pflege und in der Beziehung zu Pflegenden. Die befragten Patienten hatten zwar keine Vorstellung von der Normalstation, dennoch hatten sie aufgrund des Vergleiches mit der Intensivstation Erwartungen und waren enttäuscht, wenn diese nicht erfüllt wurden. Im Gegensatz zu Streater et al. (2001), wo gezeigt wurde, dass Angehörige die Notwendigkeit für eine intensive Pflegebeziehung aufgrund der fortschreitenden Genesung auf der Normalstation als weniger wichtig erachteten, zeigt sich, dass sie sich, unabhängig vom Genesungsfortschritt, immer eine gute Betreuung und Beziehung wünschen. Dies ist die Voraussetzung, dem Pflegeteam vertrauen zu können und sich sicher zu fühlen. Wie die Literatur zeigt, kann eine schlechte Beziehung auch zu Isolation und Verletzlichkeit führen, was sich negativ auf den Übergang und die Zeit danach auswirken kann (McKinney und Deeny 2002; Odell 2000; Wesson 1997). Pflegepersonen auf der Intensivstation nehmen bei der Transferierung eine Schlüsselposition ein, weil sie den meisten Kontakt zu den Patienten und den Angehörigen haben.

Eine gute Vorbereitung und Durchführung der Transferierung kann Relocation stress und die Angst vor der Transferierung reduzieren (Cutler und Garner 1995; Hall-Smith et al. 1997; Jones und O'Donnell 1994; National Institute for Health and Clinical Excellence 2013).

In den Ergebnissen zeigt sich auch, dass die meisten Patienten und deren Angehörige direkt oder indirekt mit unterschiedlichen Kommunikationsproblemen zu kämpfen hatten. Einerseits betraf dies die Kommunikation mit dem betreuenden Team, andererseits die Kommunikation zwischen Intensiv- und Normalstation. Im Zusammenhang damit stellt auch die erlebte Kontinuität einen weiteren, wichtigen Indikator für eine gute Übergangserfahrungen dar. Die Literatur zeigt auch, dass Pflegepersonen auf der Normalstation oft das Gefühl haben, dass viel Information verloren geht, die für die weitere Pflege eines ehemaligen Intensivpatienten notwendig ist (Lin et al. 2009; Whittaker und Ball 2000). Von Pflegenden auf beiden Seiten werden daher auch Lücken in der Versorgung identifiziert, die sich v.a. in der mangelnden Kommunikation zwischen den beiden Bereichen niederschlägt (Lin et al. 2009; Whittaker und Ball 2000).

\section{Limitationen}

Die Studie weist einige Limitationen auf. Zum einen wurden eine spezielle Gruppe an Intensivpatienten und deren Angehörigen befragt, und deshalb können die Ergebnisse nicht für alle Intensivpatienten und deren Angehörige verallgemeinert werden. Die befragten Patienten und deren An- gehörige hatten alle keine minderjährigen Kinder mehr und bis auf eine Patientin und eine Angehörige, waren alle anderen bereits in Pension. Des Weiteren wurden als wichtigste Bezugsperson aus Sicht der Betroffenen ausschließlich der Partner genannt, somit finden sich in der Untersuchung ausschließlich Paarbeziehungen, und es konnten keine anderen Konstellationen wie z.B. Geschwister, Elternteile oder erwachsene Kinder befragt werden. Die Einbeziehung dieser Gruppen von Betroffenen könnten andere bzw. ergänzende Einblicke geben.

\section{Schlussfolgerungen}

Die Ergebnisse zeigen, dass sowohl Patienten als auch Angehörige im Zusammenhang mit dem Übergang und der Zeit danach mit verschiedenen Problemen zu kämpfen haben und oft keine adäquate Unterstützung in dieser Zeit erhalten. Für viele Betroffene und auch Angehörige fehlen während des gesamten Prozesses des Übergangs von der Intensiv- auf die Normalstation und dann nach Hause eine konkrete Ansprechperson und Begleitung sowie die Erinnerung an die Zeit auf der Intensivstation. Dies stärkt die Bedarfe nach ,follow-up services“ nach einem Intensivaufenthalt bzw. eines „Liaison-nurse“-Konzepts, ausführlicher, interdisziplinär verfasster Verlegungsberichte und den Einsatz eines Intensivtagebuches. Mit deren Hilfe könnten sowohl Patienten als auch deren Angehörige besser unterstützt und Kontinuität im Übergangsprozess gewährleistet werden. Damit könnte ein Beitrag zur Kontinuität und zur Verbesserung der Qualität im Sinne der Patienten- und Angehörigenorientierung geleistet werden.

Funding Open access funding provided by University of Vienna.

Open Access Dieser Artikel wird unter der Creative Commons Namensnennung 4.0 International Lizenz (http://creativecommons.org/ licenses/by/4.0/deed.de) veröffentlicht, welche die Nutzung, Vervielfältigung, Bearbeitung, Verbreitung und Wiedergabe in jeglichem Medium und Format erlaubt, sofern Sie den/die ursprünglichen Autor(en) und die Quelle ordnungsgemäß nennen, einen Link zur Creative Commons Lizenz beifügen und angeben, ob Änderungen vorgenommen wurden.

Hinweis des Verlags Der Verlag bleibt in Hinblick auf geografische Zuordnungen und Gebietsbezeichnungen in veröffentlichten Karten und Institutsadressen neutral.

\section{Literatur}

Agard A, Egerod I, Tonnesen E, Lomborg K (2015) From spouses to caregiver and back: a grounded theory study of post-intensive care unit spousal caregiving. J Adv Nurs 71(8):1892-1903. https://doi. org/10.1111/jan.12657

Bokinskie JC (1992) Family conferences: a method to diminish transfer anxiety. J Neurosci Nurs 24(3):129-133. https://doi.org/10. 1097/01376517-199206000-00002 
Bundesministerium für Gesundheit (2012) Krankenanstalten in Zahlen. Überregionale Auswertung der Dokumentation der landesgesundheitsfondsfinanzierten Krankenanstalten 2012. Bundesministerium für Gesundheit, Wien

Cox E (2012) Persistent systemic inflammation in chronic critical illness. Respir Care 57:1021-1027

Coyle MA (2001) Transfer anxiety: Preparing to leave intensive care. Intensive Crit Care Nurs 17(3):138-143. https://doi.org/10.1054/ iccn.2001.1561

Cutler L, Garner M (1995) Reducing relocation stress after discharge from the ICU. Intensive Crit Care Nurs 11(6):333-335. https:// doi.org/10.1016/s0964-3397(95)80398-x

Duffield C, Diers D, O'Brien-Pallas L, Aisbett C, Roche M, King M, Aisbett K (2011) Nursing staffing, nursing workload, the work environment and patient outcomes. Appl Nurs Res 24:224-255

Fink MP, Suter PM (2006) The future of our specialty: Critical medicine a decade from now. Crit Care Med 34:1811-1816

Flick U, von Kardorff E, Steinke I (2008) Qualitative Forschung. Ein Handbuch, 6. Aufl. Rowohlt, Reinbeck

Gibson JME (1997) Focus of nursing in critical and acute care settings: Prevention or cure? Intensive Crit Care Nurs 13(3):163-166. https://doi.org/10.1016/s0964-3397(97)80921-4

Hall-Smith J, Ball C, Coakly J (1997) Follow-up services and the development of a clinical nurse specialist in intensive care. Intensive Crit Care Nurs 13:243-248

Jones C, O'Donnell C (1994) After intensive care - what then? Intensive Crit Care Nurs 10:89-92

Lin F, Chaboyer W, Wallis M (2009) A literature review of organizational, individual and teamwork factors contributing tot he ICU discharge process. Aust Crit Care 22(1):29-43. https://doi.org/10. 1016/j.aucc.2008.11.001

Ludin SM, Arbon P, Parker S (2013) Patients'transition in the Intensive Care Units: Concept analysis. Intensive Crit Care Nurs 29:187-192

Mayer S (2002) Der Übertritt von der Intensivstation auf die normale Abteilung. Intensivpflege 10(5):212-217. https://doi.org/10.1055/ s-2002-33722

McKinney AA, Deeny P (2002) Leaving the intensive care unit: A phenomenological study of the patients' experience. Intensive Crit Care Nurs 18:320-331
Meleis AI (2010) Transitions theory. Middle-range and situation specific theories in nursing research and practice. Springer, New York

Mitchell M, Courtney M, Coyer F (2003) Understanding uncertainty and minimizing families anxiety at the time of transfer from intensive care. Nurs Health Sci 5:207-217

Nagl-Cupal M, Schnepp W (2011) Funktion und Gestaltung familiärer Hilfen im Angesicht existenzieller Erfahrungen von Angehörigen auf der Intensivstation. Pflegewissenschaft 5:289-298

National Institute for Health and Clinical Excellence (NICE) (2013) Actually ill patients in hospital: Recognition of and response to acute illness in adults in hospital 2013. http://www.nic.org.uk/ nicemedia/live/11810/58247.pdf. Zugegriffen: 25. Nov. 2013

Odell M (2000) The patient's thoughts and feelings about their transfer from intensive care to the general ward. J Adv Nurs 31:322-329

Ramsay P, Huby G, Thompson A, Walsh T (2013) Intensive care survivors'experiences of ward-based care: Meleis'theory of nursing transitions and role development among critical care outreach services. J Clin Nurs 23:605-615

Rotondi AJ, Chelluri L, Sirio C, Mendelsohn A, Schulz R, Belle St, Im K, Donaoe M, Pinsky M (2002) Patients recollections of stressful experiences while receiving prolonged mechanical ventilation in an intensive care unit. Crit Care Med 30:746-752

Strauss AL, Corbin JM (1996) Grounded Theory. Grundlagen qualitativer Sozialforschung. Beltz, Weinheim

Streater C, Golledge J, Sutherland H, Easton J, MacDonald R, McNamara R, Looise A, Gelling L (2001) The relocation experiences of relatives leaving a neurosciences critical care unit: a phenomenological study. Nurs Crit Care 6:163-170

Wesson JS (1997) Meeting the informational, psychosocial and emotional needs of each ICU patient and family. Intensive Crit Care Nurs 13:111-118

Whittaker J, Ball C (2000) Discharge from intensive care: A view from the ward. Intensive Crit Care Nurs 16:135-143

World Medical Association (2013) World medical association declaration of Helsinki ethical principles for medical research involving human subjects. JAMA 310(20):2191-2194 